\title{
Similarity Search Algorithm for Efficient Sub-trajectory Matching in Moving Databases
}

\author{
Eun-Cheon Lim and Choon-Bo Shim* \\ Division of Information \& Communication Engineering, Sunchon National University, \\ Suncheon, 540-742, South Korea \\ \{eclim, cbsim\}@sunchon.ac.kr
}

\begin{abstract}
Similarity measure scheme on moving objects has become a topic of increasing in the area of moving databases. In this paper, we propose a new similarity search algorithm for efficient sub-trajectory matching. For measuring similarity between two sub-trajectories, we propose a new $\mathrm{v}$ (variable)-warping distance algorithm which enhances the existing time warping distance algorithm by permitting up to $\mathrm{v}$ replications for an arbitrary motion of a query trajectory. Our v-warping distance algorithm provides an approximate matching between two trajectories as well as an exact matching between them. Based on our vwarping distance algorithm, we also present a similarity measure scheme for the single trajectory in moving databases. Finally, we show that our scheme based on the $\mathrm{v}$-warping distance achieves much better performance than other conventional schemes, such as Li's one (no-warping) and Shan's one (infinitewarping) in terms of precision and recall measures.
\end{abstract}

\section{Introduction}

The most striking difference between still images and videos stems from movement and variations. Retrieving moving objects, which requires both spatial and temporal knowledge, is part of content-based retrieval on moving databases. Typical applications are : automated surveillance systems, moving object monitoring, road traffic monitoring, video on demand etc. Modeling and similarity measure schemes based on moving objects has received some research attention recently [1-2], but it is certainly in its infancy. Most research in this area focuses on tracking the movement of a moving object, i.e., the trajectory of an object over a period of time, which is certainly very important.

In this paper, we propose a new similarity search algorithm for efficient subtrajectory matching. For measuring similarity between two sub-trajectories, we propose a new $\mathrm{v}$ (variable)-warping distance algorithm which enhances the existing time warping distance algorithm by permitting up to $\mathrm{v}$ replications for an arbitrary motion of a query trajectory. Our v-warping distance algorithm provides an approximate matching between two trajectories as well as an exact matching between them. Especially in case of a large amount of moving objects, the approximate

\footnotetext{
* Corresponding author.
} 
matching can improve the performance of retrieval on moving objects' trajectories, compared with the exact matching. Based on our v-warping distance algorithm, we also present a similarity measure scheme for a moving object's trajectory in moving databases. Finally, we show that our scheme based on proposed v-warping distance achieves much better performance than other conventional schemes, such as Li's one (no-warping) and Shan's one (infinite-warping) in terms of precision and recall measures.

\section{Related Work}

There have been two main researches on retrieval based on similar sub-trajectory by measuring the similarity between a given query trajectory and data trajectories, i.e., Li's scheme and Shan's scheme. First, Li et al. [2] represented the trajectory of a moving object as eight directions, such as North(NT), Northwest(NW), Northeast(NE), West(WT), Southwest(SW), East(ET), Southeast(SE), and Southwest(SW). They represented as $\left(\mathrm{S}_{\mathrm{i}}, \mathrm{d}_{\mathrm{i}}, \mathrm{I}_{\mathrm{i}}\right)$ the trajectory of a moving object A over a given time interval $I_{i}$ where $S_{i}$ is the displacement of $A$ and $d_{i}$ is a direction. For a set of time interval $<I_{1}$, $I_{2}, \ldots, I_{n}>$, the trajectories of A can be represented as a list of motions, like $<\left(S_{1}, d_{1}, I_{1}\right)$ , $\left(\mathrm{S}_{2}, \mathrm{~d}_{2}, \mathrm{I}_{2}\right), \ldots,\left(\mathrm{S}_{\mathrm{n}}, \mathrm{d}_{\mathrm{n}}, \mathrm{I}_{\mathrm{n}}\right)>$. Based on the representation for moving objects' trajectories, they present a similarity measures to computes the similarity of spatiotemporal relationships between two moving object. Let $\left\{M_{1}, M_{2}, \ldots, M_{m}\right\}(m \geq 1)$ be the trajectory of moving object $A,\left\{N_{1}, N_{2}, \ldots, N_{n}\right\}$ be the trajectory of moving object $\mathrm{B}$, and $\mathrm{m} \leq \mathrm{n}$. The similarity measure between the trajectory of object $\mathrm{A}$ and that of object B, TrajSim(A, B), is computed by using the similarity distances of directional relations as follows. Here, $\operatorname{minDiff}(\mathrm{A}, \mathrm{B})$ and $\operatorname{maxDiff}(\mathrm{A}, \mathrm{B})$ are the smallest distance between $\mathrm{A}$ and $\mathrm{B}$ and the largest distance, respectively.

$$
\operatorname{Traj} \operatorname{Sim}(A, B)=\frac{\max \operatorname{Diff}(A, B)-\min \operatorname{Diff}(A, B)}{\max \operatorname{Diff}(A, B)}
$$

$$
(\forall j, 0 \leq j \leq n-m)
$$

Secondly, Shan and Lee [3] represented the trajectory of a moving object as a sequence of segments, each being expressed as the slope with real angle ranging from 0 to 360 degree for content-based retrieval. They also proposed two similarity measure algorithms, OCM (Optimal Consecutive Mapping) and OCMR (Optimal Consecutive Mapping with Replication), which can measure similarity between query trajectory and data trajectory. The OCM algorithm that supports exact matching measures the similarity for one-to-one segment mapping between query trajectory and data trajectory. Meanwhile, The OCMR algorithm supports approximation matching. In order to measure the similarity, each motion of query trajectory can be permitted to map with more than one motions of data trajectory.

\section{Proposed Scheme Based on v-Warping Distance Algorithm}

We first present three considerations for supporting efficient similar sub-trajectory retrieval on moving object's trajectory. 
1. The existing time warping transformation [4-5] used for a similar subsequence matching in sequence databases can allow the infinitive replication of a data sequence as well as a query sequence. However, for similar sub-trajectory retrieval in moving databases, it is necessary to allow the replication of only a query trajectory.

2. The time warping transformation for a similar sub-sequence matching can allow the infinitive replication of an arbitrary motion. However, for the similar sub-trajectory retrieval, it is necessary to support the replication of up to the variable dynamic number (v) of motions, so called v-warping distance.

3. For modeling motions being composed of the trajectory of a moving object, it is necessary to support multiple properties including angle, distance, and time, instead of the single property of angle.

The consideration 1 is generally needed for supporting an approximation matching from similar sub-trajectory retrieval and the considerations 2 and 3 are needed for improving the effectiveness of the approximation matching. In addition, the considerations 2 and 3 are very sensitive, depending on application areas. The similar subsequence matching approach which is used for the existing time warping transformation does not satisfy all of the above three considerations. The reason is why the characteristic of data used in sequence database is different from that of trajectory data of moving objects in moving databases. Generally, the sequence data has a detailed and elaborate feature and the number of elements consisting of a sequence reaches scores or hundreds. On the other hand, the trajectory data of moving objects in moving databases are composed of motions over a time interval and the number of motions consisting of a trajectory is less than scores. Meanwhile, the Shan's OCMR scheme can satisfy the considerations 1, but it does not satisfy the considerations 2 and 3.

Therefore, we propose a new dynamic v-warping distance algorithm which can support an approximation matching and satisfy the above three considerations for similar sub-trajectory retrieval. In order to satisfy the consideration 3 , we generally define the trajectory of moving objects as a collection of consecutive motions consisting of $n$-dimensional properties.

Definition 1. The trajectory of moving object $\mathrm{S}$ is defined as a set of consecutive motions, $\mathrm{S}=(\mathrm{s}[1], \mathrm{s}[2], \ldots, \mathrm{s}[|\mathrm{S}|])$, where each motion $\mathrm{s}[\mathrm{i}]$ is composed of $\mathrm{n}$ dimensional properties as follows:

$$
s[i]=(s[i, 1], s[i, 2], \ldots, s[i, n])
$$

For measuring a similarity between two trajectories, we define a v-warping distance as follows, which is newly made by applying the concept of time warping distance[45] used for time-series databases to the trajectory data of moving objects in moving databases.

Definition 2. Given two trajectory of moving objects S and Q, the v-warping distance $\mathrm{D}_{\mathrm{vw}}$ is defined recursively as follows:

$D_{\mathrm{vw}}(\mathbf{0}, \mathbf{0})=\mathbf{0}, \mathrm{D}_{\mathrm{vw}}(\mathrm{S}, \mathbf{0})=\mathbf{D}_{\mathrm{vw}}(\mathbf{0}, \mathbf{Q})=\infty$

$D_{v w}(S, Q)=D_{\text {base }}(S[1], Q[1])+\min \left(\left\{D_{v w}((S[2+i:-], Q), 0 \leq i \leq v), D_{v w}(S[2:-], Q[2:-])\right\}\right)$

$D_{\text {base }}(a, b)=d_{d f}(a, b)$ 
Our v-warping distance algorithm is shown in Figure 1. It calculates a v-warping distance between a given query trajectory $\mathrm{Q}$ and a data trajectory $\mathrm{S}$ by permitting up to $\mathrm{v}$ (variable) replications for an arbitrary motion of a query trajectory $\mathrm{Q}$. When the motions of a data trajectory and a query trajectory are represented by rows and columns in the cumulative table respectively, our a v-warping distance algorithm finds a minimum distance starting from the first column of the first row within the last column of the last row by replicating an arbitrary motion of a query trajectory up to $\mathrm{v}$ times. In addition, since a motion of a trajectory is modeled as both angle property and distance property, our algorithm measures a similarity between a data trajectory $\mathrm{S}$ and a query trajectory $\mathrm{Q}$ by considering both properties.

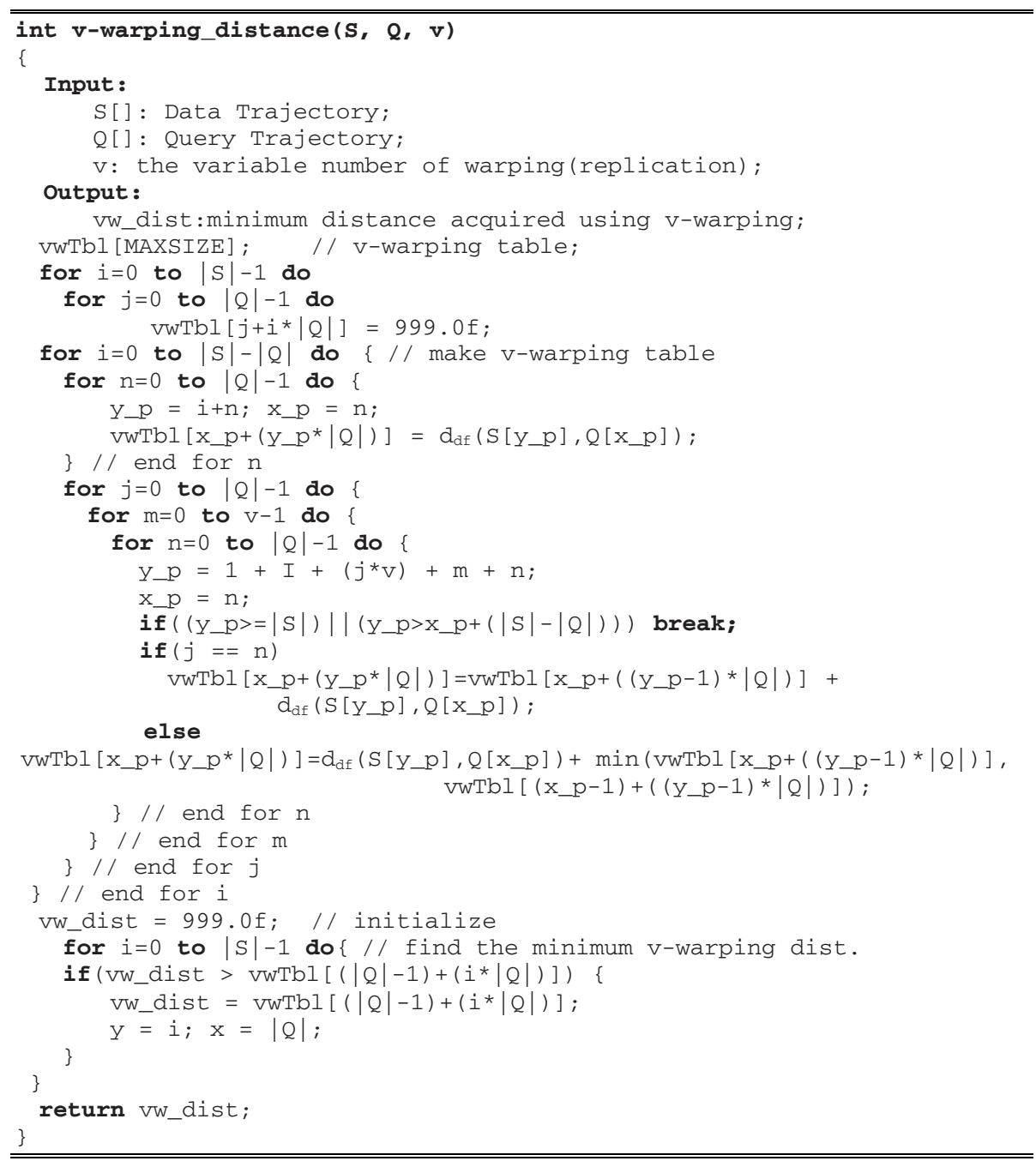

Fig. 1. Dynamic v-warping distance algorithm 


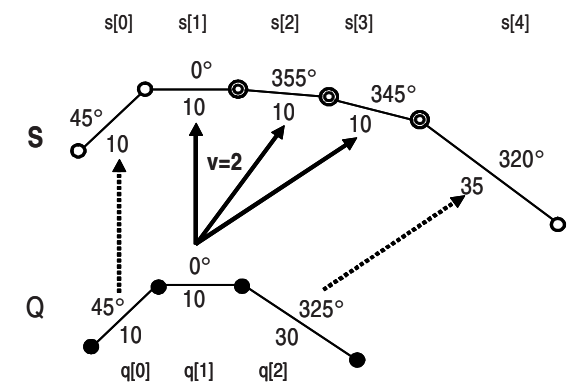

Fig. 2. Mapping of motions between $S$ and $Q$ when $v=2$

Figure 2 depicts an example of our v-warping distance algorithm which can calculate similarity between trajectory $\mathrm{S}$ and $\mathrm{Q}$ when $\mathrm{v}$ is 2 . We can permit up to $2(=v)$ times replications for an arbitrary motion of only query trajectory $Q$. In the above example, we can obtain the minimum distance value, that is, the maximum similarity value, between $\mathrm{S}$ and $\mathrm{Q}$ when $\mathrm{q}[1]$ of trajectory $\mathrm{Q}$ is mapped to each $\mathrm{s}[1]$, $\mathrm{s}[2]$, and $\mathrm{s}[3]$ of trajectory $\mathrm{S}$, instead of the exact matching, namely, one-to-one mapping between trajectory $\mathrm{S}$ and $\mathrm{Q}$. Therefore, it is shown that the approximate matching is superior to the exact mating for calculating the similarity between trajectories in moving databases.

Based on our v-warping distance algorithm, we will define a similarity measure for a single trajectory. Since we measure a similarity between i-th motion in query trajectory $\mathrm{Q}$ and $\mathrm{j}$-th motion in data trajectory $\mathrm{S}$, we define a distance function between two motions, $d_{d f}(q[i], s[j])$, as follows.

Definition 3. A distance function, $\mathrm{d}_{\mathrm{df}}(\mathrm{q}[\mathrm{i}], \mathrm{s}[\mathrm{j}])$, to measure the similarity between the arbitrary motion $\mathrm{s}[\mathrm{i}]$ of a data trajectory $\mathrm{S}$ and the arbitrary motion $\mathrm{q}[\mathrm{j}]$ of a query trajectory Q is defined as follows.

$$
\begin{aligned}
& \mathrm{d}_{\text {dis }}(\mathrm{s}[\mathrm{i}, 2], \mathrm{q}[\mathrm{j}, 2])=|\mathrm{s}[\mathrm{i}, 2]-\mathrm{q}[\mathrm{j}, 2]| \\
& \text { if }|\mathrm{s}[\mathrm{i}, 1]-\mathrm{q}[\mathrm{j}, 1]|>180 \text { then } \mathrm{d}_{\text {ang }}(\mathrm{s}[\mathrm{i}, 1], \mathrm{q}[\mathrm{j}, 1])=(360-|\mathrm{s}[\mathrm{i}, 1]-\mathrm{q}[\mathrm{j}, 1]|) \\
& \quad \text { else } \mathrm{d}_{\text {ang }}(\mathrm{s}[\mathrm{i}, 1], \mathrm{q}[\mathrm{j}, 1])=|\mathrm{s}[\mathrm{i}, 1]-\mathrm{q}[\mathrm{j}, 1]| \\
& \mathrm{d}_{\mathrm{df}}(\mathrm{s}[\mathrm{i}], \mathrm{q}[\mathrm{j}])=\left(\left(\left(\mathrm{d}_{\text {ang }} / 180\right) * \alpha\right)+\left(\left(\mathrm{d}_{\mathrm{dis}} / 100\right) * \beta\right)\right)
\end{aligned}
$$

Here, $d_{\text {ang }}$ is a distance function for the direction (angle) property for all the motions of a trajectory and $d_{d i s}$ is a distance function for the distance property. $\mathrm{s}[\mathrm{i}, 1]$ and $\mathrm{s}[\mathrm{i}$, 2] are the direction and the distance value of the i-th motion in a trajectory $S$, respectively. $\alpha$ and $\beta$ mean the weight of the direction and the distance, respectively, when $\alpha+\beta=1.0$.

For example, by using our v-warping distance algorithm, a similarity distance between a data trajectory $S=\{(45,10),(0,10),(355,10),(345,10),(4,40),(325,45)\}$ and a query trajectory $\mathrm{Q}=\{(45,10),(0,10),(325,10)\}$ can be calculated in Figure 3 . The value of the last column of the last row means the minimum distance 0.30 by permitting the infinitive replications of the query trajectory $Q$ as shown in trajectory $\mathrm{S} 1$. In the case of $\mathrm{v}$-warping distance, the motion of $\mathrm{q}[0]$ in the query trajectory $\mathrm{Q}$ corresponds to the $s[0]$ in the data trajectory $S$, the motion of $q[1]$ to the $s[1]$, the motion of $\mathrm{q}[1]$ to the $\mathrm{s}[2]$, and the motion of $\mathrm{q}[2]$ to the $\mathrm{s}[3]$ respectively as shown in 
trajectory S2. Finally, we can find a path starting from the first column of the first row within the last column of the last row, thus obtaining the minimum distance by permitting up to $\mathrm{v}(=2)$ replications. We can summarize the differences of distance between each motion of the query and the data trajectory on the path, that is, $\mathrm{q}[0]-$ $\mathrm{s}[0]|+| \mathrm{q}[1]-\mathrm{s}[1]|+| \mathrm{q}[1]-\mathrm{s}[2]|+\mathrm{q}[2]-\mathrm{s}[3]|=0.00+0.00+0.02+0.07=0.09$. This is a minimum distance value between the two trajectories by using our v-warping distance algorithm. Thus, the similarity degree between $\mathrm{S}$ and $\mathrm{Q}$ is $91 \%(=1-0.09)$ while the similarity degree based on Shan's OCMR(infinite warping) is 70\%(=1-0.30). In conclusion, our similarity measure scheme based on the v-warping distance algorithm provides a better result than Shan's OCMR.

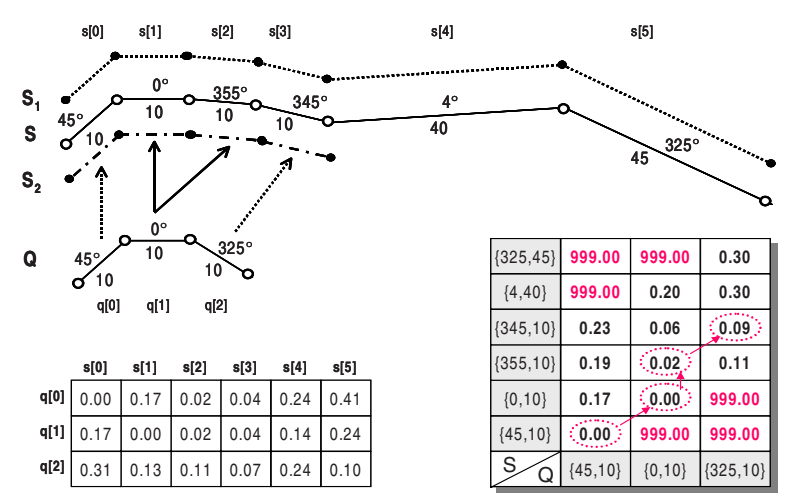

Fig. 3. Example of similarity measure between $\mathrm{S}$ and $\mathrm{Q}(\mathrm{v}=2)$

\section{Experimental Results}

In order to verify the usefulness of our representation scheme for moving object's trajectory, we do the performance analysis by using real soccer video data. Since soccer video data have many trajectories of soccer balls, i.e., salient objects, it is necessary to extract the trajectories of moving objects from the soccer ball. Most of video data, formatted as MPEG file, which are used in our experiment include a shot of 'getting a goal'. We extract the trajectories of a soccer ball by manually tracing the ball in a ground field. For our experiment, we make 50 query trajectories consisting of twenty in 'the right field' and twenty in 'the left field' from the half line of the ground field. The experimental data used for performance evaluation is as follows: (1) data domain : trajectory extracted from real soccer video data; (2) salient moving object : soccer ball; (3) data set : 1000 ; (4) the average number of motions for trajectory : 9.1 ; (5) the number of query : 50.

We implemented our similarity search algorithm under Windows 2003 Server O.S with Pentium Processor and 1 GB memory by using Microsoft Visual C++ compiler. We compare our representation scheme with the Li's and Shan's schemes in terms of retrieval effectiveness, that is, average precision and recall measures. Let RD (Relevant data in Database) be the number of video data relevant to a given query which are selected from the database, RQ (Retrieved data by Query) be the total 
number of data retrieved by a given query, and RR (Relevant data that are Retrieved) be the number of relevant data retrieved by a given query. In order to obtain RD, we make a test panel which selects relevant data manually from the database. The precision is defined as the proportion of retrieved data being relevant and the recall is defined as the proportion of relevant data being retrieved as follows.

$$
\text { Precision }=\frac{R R}{R Q} \quad \text { Recall }=\frac{R R}{R D}
$$

For our performance comparison, we adopt the 11-point measure [6], which is most widely used for measuring the precision and recall. For a moving object's trajectory, we consider the weight of angle $\left(\mathrm{W}_{\mathrm{a}}\right)$ and the weight of distance $\left(\mathrm{W}_{\mathrm{d}}\right)$ separately since we use both angle and distance for modeling the trajectory of moving objects. We also take into account the number of replications (v) since $\mathrm{v}$ is a very important parameter, depending on an application area. Here we do our experiment when $v=0$, 1 , and 2 owing to the characteristics of the trajectory of the soccer ball in soccer video data. $v=0$ is exact matching and $v=1$ and 2 is approximate matching. We show from our experiment that there is no difference on retrieval effectiveness when $\mathrm{v}$ is greater than 2. Table 1 shows the retrieval effectiveness of our scheme, Li's scheme, and Shan's scheme. In case we do our performance analysis based on only the angle property $\left(\mathrm{W}_{\mathrm{a}}=1.0\right.$ and $\left.\mathrm{W}_{\mathrm{d}}=0.0\right)$, it is shown that our scheme achieves about $10-15 \%$ higher precision than that of Li's and Shan's schemes while it holds about the same recall. In case we consider the weight of angle about two times greater than that of distance $\left(\mathrm{W}_{\mathrm{a}}=0.7\right.$ and $\left.\mathrm{W}_{\mathrm{d}}=0.3\right)$, it is shown that our scheme achieves about $15-20 \%$ higher precision than that of Li's and Shan's schemes while it holds about the same recall.

Table 1. Experimental results

\begin{tabular}{|c|c|c|c|c|c|c|c|}
\hline & & \multicolumn{3}{|c|}{ Avg. Precision } & \multicolumn{3}{|c|}{ Avg. Recall } \\
\hline & \# of warping & $\mathrm{v}=\mathbf{0}$ & $\mathrm{v}=1$ & $\mathrm{v}=2$ & $\mathrm{v}=1$ & $\mathrm{v}=1$ & $\mathrm{v}=\mathbf{2}$ \\
\hline \multirow{3}{*}{$\begin{array}{c}W_{\mathrm{a}}: W_{\mathrm{d}}= \\
1.0: 0.0\end{array}$} & Li's Scheme & \multicolumn{3}{|c|}{0.25} & \multicolumn{3}{|c|}{0.45} \\
\hline & Shan's Scheme & \multicolumn{3}{|c|}{0.30} & \multicolumn{3}{|c|}{0.44} \\
\hline & Our Scheme & 0.34 & 0.38 & 0.40 & 0.51 & 0.48 & 0.47 \\
\hline \multirow{3}{*}{$\begin{array}{c}W_{\mathrm{a}}: \mathrm{W}_{\mathrm{d}}= \\
0.7: 0.3\end{array}$} & Li's Scheme & \multicolumn{3}{|c|}{0.25} & \multicolumn{3}{|c|}{0.45} \\
\hline & Shan's Scheme & \multicolumn{3}{|c|}{$\begin{array}{l}\mathbf{0 . 3 0} \\
\end{array}$} & \multicolumn{3}{|c|}{0.44} \\
\hline & Our Scheme & 0.39 & 0.44 & 0.45 & 0.50 & 0.46 & 0.47 \\
\hline \multirow{3}{*}{$\begin{array}{c}W_{\mathrm{a}}: W_{\mathrm{d}}= \\
0.5: 0.5\end{array}$} & Li's Scheme & \multicolumn{3}{|c|}{0.25} & \multicolumn{3}{|c|}{0.45} \\
\hline & Shan's Scheme & \multicolumn{3}{|c|}{0.30} & \multicolumn{3}{|c|}{0.44} \\
\hline & Our Scheme & 0.33 & 0.34 & 0.38 & 0.51 & 0.50 & 0.51 \\
\hline
\end{tabular}

From our experiment, we finally show that our similarity measure scheme based on our v-warping distance algorithm achieves better performance on average precision than Li's and Shan's schemes while it holds about the same recall in the moving object's trajectory. Particularly, the performance of our scheme is the best when the weight of angle is over two times than that of distance $\left(\mathrm{W}_{\mathrm{a}}=0.7\right.$ and $\left.\mathrm{W}_{\mathrm{d}}=0.3\right)$. 


\section{Conclusions}

In this paper, we propose a new similarity search algorithm for efficient sub-trajectory matching. For measuring similarity between two sub-trajectories, we propose a new v-warping distance algorithm which enhances the existing time warping distance algorithm by permitting up to $\mathrm{v}$ replications for an arbitrary motion of a query trajectory. Our v-warping distance algorithm provides an approximate matching between two trajectories as well as an exact matching between them. From our experiment, we showed that our similarity measure scheme based on the v-warping distance outperformed Li's and Shan's schemes in terms of precision and recall measures. The performance of our scheme achieves about $15-20 \%$ performance improvement against Li's one and Shan's one when the weight of angle is over two times greater than that of distance.

\section{Acknowledgements}

This work was supported by the Korea Research Foundation Grant funded by the Korean Government (MOEHRD) (KRF-2006-331-D00461).

\section{References}

1. Forlizzi, L., Guting, R. H., Nardelli, E., Schneider, M.: A Data Model and Data Structures for Moving Objects Databases, Proc. of ACM SIGMOD Conf, (2000)319-330

2. Li, J., Ozsu, M. T., Szafron, D.: Modeling Video Temporal Relationships in an Object Database Management System," in Proceedings of Multimedia Computing and Networking(MMCN97), (1997)80-91

3. Shan, M., Lee, S. Y.,: Content-based Video Retrieval via Motion Trajectories, in Proceedings of SPIE Electronic Imaging and Multimedia System II, Vol. 3561, (1998)52-61

4. Yi, B., Lagadish, H. V., Faloutsos, C.: Efficient Retrieval of Similar Time Sequences Under Time Warping, In Proc. Int'l. Conf. on Data Engineering, IEEE,( 1998)201-208

5. Park, S. H. et al.: Efficient Searches for Simialr Subsequence of Difference Lengths in Sequence Databases, In Proc. Int'l. Conf. on Data Engineering. IEEE, (2000)23-32

6. Salton, G., McGill, M.: An introduction to Modern Information Retrieval, McGraw-Hill, (1993) 